\title{
Immune Dysfunction in Patients with Chronic Lymphocytic Leukemia and Challenges during COVID-19 Pandemic
}

\author{
Petra Langerbeins ${ }^{a, b}$ Barbara Eichhorst ${ }^{a, b}$ \\ aDepartment I of Internal Medicine, Center for Integrated Oncology Aachen Bonn Cologne Düsseldorf, Cologne, \\ Germany; ${ }^{\mathrm{b} C L L}$ Study Group, University Hospital Cologne, University of Cologne, Cologne, Germany
}

\section{Keywords}

Chronic lymphocytic leukemia · Coronavirus disease 2019 .

Infections $\cdot$ Immunodeficiency

\begin{abstract}
The novel severe acute respiratory syndrome coronavirus (SARS-CoV-2) has been first described in December 2019 in Wuhan, China, and has led to a worldwide pandemic ever since. Initial clinical data imply that cancer patients are particularly at risk for a severe course of SARS-CoV-2. In patients with chronic lymphocytic leukemia (CLL), infections are a main contributor to morbidity and mortality driven by an impaired immune system. Treatment initiation is likely to induce immune modulation that further increases the risk for severe infections. This article aims to give an overview on pathogenesis and risk of infectious complications in patients with CLL. In this context, we discuss current data of SARSCoV-2 infections in patients with CLL and how the pandemic impacts their management.

(c) 2021 S. Karger AG, Basel
\end{abstract}

\section{Introduction}

Chronic lymphocytic leukemia (CLL) is the most common form of leukemia with an incidence of 4.2:100,000 per year. The median age at initial diagnosis is 70 years, and so far there is no curative therapy for the disease except for allogeneic stem cell transplantation [1]. CLL patients represent a large subgroup of patients in practices and hospitals. In the treatment-free intervals, CLL patients regularly present themselves for follow-up examinations with blood count control and physical examination in order to detect symptomatic disease progression in time. Patients receiving CLL treatment come at regular intervals for treatment administration, for example, antibody therapy, chemotherapy, or controlled dosing of targeted therapies, as well as for monitoring treatment tolerability and assessing treatment response.

Infections are the main cause of death in CLL patients. Pathogenesis is multifactorial including disease- and treatment-related factors resulting in defects in cell-mediated immunity, complement, neutrophil, or phagocytic function and antibody production.

The severe acute respiratory syndrome coronavirus 2 (SARS-CoV-2) has infected $>50$ million people and is as- 
Table 1. Deficiencies in the adaptive and innate immune response in patients with CLL

Adaptive immune response

T-cell defects
CLL-specific immune response with increased mobilization of T cells

Functional T-cell deficiency with impaired cell-to-cell contact

T-cell inhibition by CLL cells

B-cell defects

CLL-related inhibition of antibody-producing cells leading to hypogammaglobulinemia Decreased number of healthy non-CLL B cells

Innate immune response

Defects of the complement system Decreased levels of complement in majority of patients with CLL

Functional complement deficiency with inability to coat bacterial pathogens

Phagocytic cell defects

Neutrophils Impaired bactericidal activity of neutrophils

Reduction in C5a-induced chemotaxis

Monocytes Increased number of monocytes in patients with CLL

Expression of nonclassical phenotype with weak phagocytosis and impaired cytokine release

Natural killer cells

Increased number of natural killer cells in patients with CLL

Impaired cytotoxicity due to defective expression of coreceptors

CLL, chronic lymphocytic leukemia.

sociated with $>1,000,000$ deaths worldwide. Cancer patients have been identified to be at particular risk for a severe course of the coronavirus disease 2019 (COVID19) [2-5]. The following is a brief summary of CLL-related immune dysfunction, the current evidence of COVID-19 in the context of CLL, and how it impacts management of patients with CLL.

\section{CLL-Related Immune Dysfunction}

Dysregulation of the innate and adaptive immune system is a key feature in CLL patients, which is already existing in early CLL disease stages and worsening during the course of disease. These dysregulations are summarized in Table 1.

Deficiencies in the adaptive immune response include $\mathrm{T}$-cell defects and hypogammaglobulinemia. $\mathrm{T}$ cells are often increased at early stages and show progressive accumulation and exhaustion in CLL patients with advanced disease stages [6]. The mechanism is unknown but may relate to increased mobilization from secondary lymphoid tissue, or to an absolute increase of T-cell numbers which may be interpreted as a CLL-specific immune response. Despite the numeric change of T cells, there is evidence of functional deficiency of T cells with impaired cell-to-cell contact that dampens the immune response [7-10].

Management of CLL during Pandemic
Hypogammaglobulinemia affects up to $85 \%$ of all patients with CLL and may affect all immunoglobulin subclasses (IgG, IgA, and IgM) [11]. Lower immunoglobulin levels correlate with increased risk for severe and recurrent infections and the severity increases with progression of disease [12]. Possible mechanism is inhibition of antibody-producing cells by CLL cells [13].

Additionally, the percentage of healthy B cells is significantly decreased in the peripheral blood of patients with CLL [14-16]. Deficiencies of the innate immune response include defects in the complement system as well as deficiencies in neutrophils, monocytes, and dendritic and natural killer cells.

Reduced levels of complement occur in up to $40 \%$ of all patients with CLL. Despite the quantitative change, complement components also show functional impairment in patients with CLL linked to an increased risk of infections [17]. The inability to coat bacterial pathogens with C3b may be the main contributor [17].

Neutrophil deficiencies include impaired bactericidal activity and a reduction in C5a-induced chemotaxis [18, 19]. Neutropenia due to bone marrow involvement further increases the risk of severe and prolonged infections.

The total number of monocytes and natural killer cells is increased in patients with CLL, but the cells bear a range of qualitative deficiencies. Monocytes carry a nonclassical phenotype and have a gene expression profile associated 
with immunosuppressive properties. Nonclassical monocytes are weak phagocytes that do not produce reactive oxygen species or cytokines and react poorly to lipopolysaccharide [20]. Natural killer cells are impaired cytotoxic possibly primarily because of defective expression of the NKG2D coreceptor [21].

Dendritic cells from patients with active CLL show an incomplete degree of maturation and have a reduced ability to release interleukin 12 . The dendritic cell compartment is therefore unable to stimulate an effective T-cell response [22]. The defect features of dendritic cells disappear once the CLL patient has a remission [23].

\section{Supportive Care in Immunocompromised Patients with CLL}

Supportive treatment in immunodeficient CLL patients includes early vaccination and prophylactic IV immunoglobulin (IVIG) substitution.

\section{Vaccination}

Although vaccination against Streptococcus pneumoniae and seasonal influenza is recommended for patients with CLL, the protective effect of vaccination remains questionable [24]. Besides the 23 pneumococcal polyvalent vaccine (PPSV23) already certified since 1983, a second conjugate vaccine (PCV13) is available $[25,26]$. PCV13 contains small amounts of polysaccharides extracted from the capsule that surrounds the S. pneumoniae bacterium. These polysaccharides have been purified and then conjugated to a carrier. The vaccine is also adsorbed onto an aluminum compound to enhance the immune response studies with clinical endpoints which were only conducted in AIDS patients in whom PPSV23 showed no protective effect. For other forms of immunodeficiency or immunosuppression, only immunogenicity studies are available, in which PCV13 partially induced higher antibody levels against the 12 common serotypes than PPSV23. However, a correlation of higher antibody levels with better clinical protective effect is not proven. Due to the possibly better protective effect of PCV13 against the serotypes contained in the vaccine in this risk group on the one hand and the broader serotype coverage of PPSV23 on the other hand, individuals with congenital or acquired immune defects or immunosuppression should be vaccinated sequentially with PCV13 followed by PPSV23.

Clinical data indicate that in ibrutinib-treated patients, the serological immune response is impaired [27, 28].
Therefore, patients should be vaccinated as early as possible since evidence shows that vaccination at an early disease stage (before commencement of treatment and onset of hypogammaglobulinemia) is most beneficial [29-31].

\section{Immunoglobulin Substitution}

Several phase III studies conducted in the 1980s and 1990s have shown that prophylactic use of IVIG decreases bacterial infection rates in CLL patients with severe hypogammaglobulinemia [12, 32-34]. IVIG substitution should be applied to patients meeting the European Medicines Agency criteria for immunoglobulin substitution (recurrent infections in patients with $\operatorname{IgG}<4 \mathrm{~g} / \mathrm{L}$ due to secondary immunodeficiency) [35].

\section{Treatment-Related Immune Dysfunction in CLL}

In contrast to other hematologic diseases, patients with CLL usually do not require immediate treatment when diagnosed. Asymptomatic patients are observed (watch-and-wait strategy) until treatment indication according to the International Workshop on CLL criteria (IWCLL) are met [36].

Due to the development of targeted treatments attacking the B-cell receptor (BCR) pathway or defective apoptotic mechanisms in CLL, chemotherapy-based regimen is less frequently used in this disease now. However, also targeted agents are associated with risk of infections.

\section{Chemoimmunotherapy}

With the introduction of antibody-based combination therapy in 2008, a significant survival benefit for patients with CLL was demonstrated for the first time, and the chemoimmunotherapy fludarabine, cyclophosphamide, and rituximab received approval for patients in need of treatment [37]. Until today, patients with a mutated IGHV status and presence of other favorable genetic aberrations only benefit from chemoimmunotherapy with a progression-free survival of $>10$ years [38].

Alkylating agents (e.g., cyclophosphamide, chlorambucil, and bendamustine) are associated with myelosuppression and bacterial infections including pneumonia, urinary tract infections, and bacteremia caused by Staphylococcus aureus, Streptococcus pneumonia, Haemophilus influenzae, or Klebsiella species plurales (spp.) [39, 40]. Purine analogs such as fludarabine interfere with DNA 
synthesis, depleting CD4+ T cells and decreasing B cells and monocytes. Consequently, opportunistic infections with herpes simplex virus, varicella-zoster virus, or Candida spp. are commonly observed. Anti-CD20 antibodies bind CD20, inducing apoptosis and B-cell depletion persisting for up to 24 months after treatment discontinuation. Viral infections and reactivations (e.g., hepatitis B reactivation or JC-virus infection) are a known infectious complication after B-cell depleting therapy $[41,42]$.

\section{BTK-Inhibitors}

The analysis of the role and function of the BCR with its signal transduction pathway for CLL proliferation led to the development of BCR inhibitors (Bruton's tyrosine kinase inhibitors [BTKis] such as acalabrutinib or ibrutinib and PI3K inhibitors such as idelalisib or duvelisib) [43-47]. Outside clinical trials, BCR inhibitors are intended as a long-term therapy with excellent disease control. Daily intake of medication requires high patient compliance.

BCR inhibitors are now preferably used as first-line and relapse treatment of patients with CLL [43-45]. In the majority of patients, targeted drugs are well tolerated. Initial reports indicated recovery of humoral immune function as described by Sun et al. [48] in patients treated with ibrutinib. The group investigated the impact of ibrutinib on immunoglobulin levels, normal B cells, and infection rate in CLL patients in a phase 2 trial. Consistent with previous reports, immunoglobulin $G$ levels remained stable during the first 6 months on treatment, but decreased thereafter. In contrast, there was a transient increase in IgM and a sustained increase in IgA levels. Patients with superior immune reconstitution, as defined by an increase in serum IgA of $\geq 50 \%$, had a significantly lower rate of infections indicating that ibrutinib allows a clinically meaningful recovery of humoral immune function.

However, with longer follow-up data available, there have also been increasingly reports of severe bacterial and fungal infections in patients treated with ibrutinib $[49,50]$. Opportunistic infections typically occur in patients with acquired CD4 T-cell immune defects (HIV/ AIDS), but are rare in patients with genetic BTK deficiency suffering from X-linked agammaglobulinemia [51]. Patients with CLL are generally considered at low risk for invasive fungal infections [52]. However, opportunistic infections with Pneumocystis jirovecii, Cryptococcus, and Aspergillus spp. have been reported in a number of patients treated with ibrutinib either alone or in

Management of CLL during Pandemic combination with other immune modulatory drugs [49, $53,54]$. Ibrutinib is an irreversible inhibitor of BTK and IL2-inducible T-cell kinase $[55,56]$. BTK is expressed in all bone marrow cell lineages, with the exception of $\mathrm{T}$ cells and plasma cells [57]. It has been shown that ibrutinib has off-target effects on other kinases effecting CD4 $\mathrm{T}$-cell function [58]. In addition, a direct inhibitory effect of ibrutinib on BTK signaling in monocytes and macrophages could affect susceptibility to invasive fungal infections $[59,60]$. In particular, BTK is involved in triggering the receptor expressed on myeloid cells 1 (TREM1 and CD354) signaling in monocytic cells and an important inflammatory receptor that seems to be involved in the pathogenesis of infectious complications [59]. Fiedler et al. [61] demonstrated in a mouse model of X-linked agammaglobulinemia that BTK is an important regulator of neutrophilic granulocyte maturation and function in vivo. Furthermore, ibrutinib inhibits platelet adhesion to lymphatic endothelial cells by offtarget binding of SRC kinases. By this mechanism, also platelet internalization of Aspergillus spp. is inhibited which might also contribute to increased susceptibility for invasive fungal infections [62].

\section{BCL-2 Inhibitors}

A further substance class, BCL-2 inhibitors (venetoclax), increases the apoptosis of CLL cells by selective inhibition of the overexpressed BCL-2 protein. These new orally administered substances are effective and well tolerated. They are approved as monotherapy or in combination therapy with antibodies inducing high rates of minimal residual negative remissions [63-65]. The BCL2 inhibitor venetoclax induces rapid apoptosis of CLL cells, which are known to be BCL-2 dependent. Venetoclax inhibits granulopoiesis in vitro and in animal models [66]. More than $30 \%$ of treated patients develop severe CTC grade 3-5 neutropenia during the course of treatment $[64,67]$. Neutropenia occurs mainly within the first 3 cycles of treatment. CLL-associated neutropenia is likely to contribute to this toxicity particularly in the beginning of treatment. Neutropenia is usually managed with either dose tapering of venetoclax (dose reduction or interruption) or granulocyte colony-stimulating factor stimulation. The overall rate of grade 3 or higher infections is approximately $20 \%$ and comparable to chemoimmunotherapy $[63,65,68]$.

De Weerdt et al. [69] explored the effects of venetoclax on nonleukemic cells in peripheral blood and lymph 
Table 2. SARS-CoV-2 in patients with CLL - current data

\begin{tabular}{|c|c|c|c|c|}
\hline Scarfò et al. [71] & 190 & 32.5 & Age $\geq 65$ years & BTKis appeared to have a protective effect \\
\hline Mato et al. [72] & 198 & 33 & $\begin{array}{l}\text { Increased age } \\
\text { Comorbidity } \\
\text { Asthma } \\
\text { Chronic renal disease }\end{array}$ & Treatment exposure had no impact on survival \\
\hline Paneesha et al. [73] & 4 & 75 & $\begin{array}{l}\text { Comorbidity } \\
\text { Age }\end{array}$ & Treatment-naïve patients only were included \\
\hline Fürstenau et al. [74] & 7 & 29 & $\begin{array}{l}\text { Hypogammaglobulinemia } \\
\text { Impaired T-cell function }\end{array}$ & $\begin{array}{l}\text { Physically fit patients receiving venetoclax-based } \\
\text { treatment were analyzed }\end{array}$ \\
\hline Martín-Moro et al. [75] & 6 & 0 & Active disease & $\begin{array}{l}\text { Patients with no active cancer (watch and wait and } \\
\text { remission status) had better outcome }\end{array}$ \\
\hline
\end{tabular}

CLL, chronic lymphocytic leukemia; BTKi, Bruton's tyrosine kinase inhibitor.

nodes. The group demonstrated that the absolute numbers of B-, T-, and NK-cells as well as the overproduction of inflammatory cytokines were reduced. Furthermore, a restoration of NK-cell function was observed. These data support the notion that venetoclax-based regimens reduced the immunosuppressive footprint of CLL, suggesting immune recovery after the elimination of leukemic cells.

To mitigate the risk of tumor lysis syndrome, patients start venetoclax treatment in a weekly ramp-up. If the patient has an increased risk for tumor lysis according to the Cairo-Bishop definition, hospital admission might be indicated for the ramp-up phase [70].

\section{SARS-CoV-2 in Patients with CLL}

Considering the complex immune dysfunction, an increased risk for a severe course of SARS-CoV-2 infection can easily be assumed - both for untreated and treated patients with CLL. One of the largest retrospective studies by the European Research Initiative on CLL (ERIC) identified 190 patients with CLL plus confirmed SARS-CoV-2 infection [71]. Almost $80 \%$ of the patients presented with a severe course of COVID-19. Severity was defined by need of oxygen and/or intensive care admission. The mortality rate of hospitalized patients was $32.5 \%$. Risk factors for a severe course with respiratory distress were an advanced age of $\geq 65$ years - but age did not impact mortality. Surprisingly, only $39.7 \%$ of infected patients were receiving or had recently been receiving treatment for CLL. Patients on recent or ongoing treatment were more likely to have a milder COVID-19 compared to those with severe disease who in the vast majority were on a watch-and-wait follow-up. When comparing administered CLL treatment, BTKis appeared to have a protective effect in terms of severity of COVD-19 measured by hospitalization rate. Hypogammaglobulinemia and comorbidity did not show a relevant impact on the clinical course of COVID-19 patients (Tables 2, 3).

Another large case series collected information on 198 CLL patients with COVID-19 from 20 US centers and 23 international centers in the European Union/United Kingdom and South America [72]. The mortality rate observed here with $33 \%$ of all hospitalized patients (178 patients) was similar to that in the ERIC cohort. In spite of the fact that in this cohort $61 \%$ of patients had prior or were on current CLL therapy, treatment exposure did not have here an impact on survival. The watch-and-wait and treated cohorts had similar rates of admission (89 vs. $90 \%$ ), intensive care unit admission ( 35 vs. $36 \%$ ), intubation ( 33 vs. $25 \%$ ), and mortality ( 37 vs. $32 \%$ ). The results of this retrospective analysis did not confirm the beneficiary effect of a BTKi treatment compared to a non-BTKi treatment on survival (case fatality rate, 34 vs. $35 \%$ ), though the BTKi was held during the COVID-19 course for the majority of patients. In multivariable analyses, significant predictors for an adverse outcome of COVID-19 were increased age, comorbidity, asthma, and chronic renal disease. 
Table 3. Summary of clinical management of patients with CLL during COVID-19 pandemic

Vaccinate against S. pneumonia and seasonal influenza as early as possible

Substitute immunoglobulins in patients meeting the European Medicines Agency criteria for immunoglobulin substitution (35)

Continue watch-and-wait strategy in asymptomatic CLL patients lacking treatment indication

Try to decrease number of visits in stable CLL patients during pandemic peak

Exclude COVID-19 before initiating treatment by careful anamnesis, examination, and PCR testing of the nasopharynx

Chose CLL treatment based on the individual patients' risk profile including medical history (age, comorbidities, previous lines of treatment, and concomitant medication) and genetic and laboratory risk factors (TP53-status, deletion 17p, and IgHV mutational status)

Initiate treatment depending on epidemic trajectory and local situation of the hospital

Use G-CSF in treatment-associated neutropenia

Cautiously stop treatment in case of symptomatic SARS-CoV-2 infection and hold treatment until the patient is fully recovered

CLL, chronic lymphocytic leukemia; G-CSF, granulocyte colony-stimulating factor.

A small case series by Paneesha et al. [73] reported 4 treatment-naive CLL patients with a severe course of COVID-19 resulting in a high mortality rate of $75 \%$. Following the before-mentioned reports, comorbidity and age were predominant in the fatal cases with 3 patients being roundabout 80 years of age whilst the surviving patient was rather young (49 years).

The immunomodulatory role of venetoclax-based combinations and chemoimmunotherapy in the context of SARS-CoV-2 was analyzed by Fürstenau et al. [74]. Within a prospective phase 3 trial, 7 physically fit patients with CLL developed COVID-19. All but 1 patient had received venetoclax-based combination treatment. Six of 7 patients had to be hospitalized, and 2 patients died as a result of their SARS-CoV-2 infection. History of hypogammaglobulinemia, as well as an impaired T-cell function, was documented in the majority of patients. This case series underscores that even young and fit patients with CLL are at increased risk for a complicated or lethal course of SARS-CoV-2.

A retrospective single-center analysis of patients with hematological malignancies who developed COVID-19 by Martín-Moro et al. [75] identified 6 CLL patients that had been hospitalized due to SARS-CoV-2 infection. In this small case series, patients with lymphoproliferative disorders (including CLL) had a superior survival compared to plasma cell dyscrasias, acute leukemias, myeloproliferative neoplasms, and myelodysplastic syndromes. Patients with no active cancer (watch-and-wait strategy and remission status) presented better outcomes.

Management of CLL during Pandemic
The Hospital Clinic from Barcelona provided an attempt to determine the prevalence of COVID-19 in patients with CLL by analyzing 2,366 hospitalized patients infected with SARS-CoV-2 during the first peak of the pandemic. Among those were 4 CLL patients with a mild course measured by admission to intensive care [76]. The here calculated prevalence of SARS-CoV-2 infections among CLL patients was rather low with $0.95 \%$ ( 4 of 420 patients, representative of the general CLL population).

To investigate the potential role of ongoing CLL treatment on the outcome of patients infected by SARSCoV-2, the CLL commission of the Lombardy Hematology Network analyzed data from 2902 patients with CLL treated with ibrutinib $(N=278)$, venetoclax $(N=50)$, idelalisib $(N=9)$, and chemoimmunotherapy $(N=18)$ [77]. A total of 23 SARS-CoV-2 infections were identified, of whom 15 patients did not receive any treatment. The remaining patients received ibrutinib $(N=4)$, venetoclax $(N=3)$, and idelalisib $(N=1)$. The authors conclude that the low incidence of COVD-19 positivity among ibrutinib-treated patients ( 4 of 278) might hint to the modulating immune activity of ibrutinib, possibly mitigating clinical manifestations of infection-driven cytokine release syndrome.

\section{COVID-19 Treatments in Patients with Cancer}

So far, the nucleotide analog remdesivir is the only registered drug to treat SARS-CoV-2 and shortens time until recovery in hospitalized patients without reduction of 
mortality [78]. The RECOVERY UK study investigated the use of intravenous dexamethasone (at a dose of $6 \mathrm{mg}$ once daily) in patients who were hospitalized with $\mathrm{CO}$ VID-19 [79]. Preliminary results of this study demonstrated a lower 28-day mortality rate among those who were receiving either invasive mechanical ventilation or oxygen alone at randomization but not among those receiving no respiratory support.

Two large retrospective studies of COVID-19 and Cancer Consortium (CCC19) evaluated the impact of COVID-19 treatment in patients with cancer. The first study by Kuderer et al. [80] reported a 30-day all-cause mortality of $13 \%$ among patients with active or prior cancer and confirmed SARS-CoV-2 infection and suggested increased 30-day all-cause mortality among patients receiving the combination of hydroxychloroquine plus azithromycin. Other factors associated with increased mortality included age, male sex, former smoking status, number of comorbidities, Eastern Cooperative Oncology Group performance status (ECOG PS) of 2 or higher, and active cancer.

The follow-up study by Rivera et al. [81] examined the association of COVID-19 treatments with 30-day allcause mortality among 2,186 patients with cancer and confirmed SARS-CoV-2 infection. Hydroxychloroquine with any other drug was associated with increased mortality versus treatment with any COVID-19 treatment other than hydroxychloroquine or untreated controls. Remdesivir had numerically reduced mortality versus untreated controls that did not reach statistical significance. The encouraging findings for corticosteroids in the abovementioned UK RECOVERY trial were not replicated in this cohort of patients with cancer [79].

\section{Convalescent Plasma for the Treatment of Severe COVID 19 Infection in Cancer Patients}

Passive immunization is the transfer of virus-neutralizing antibodies that are contained in convalescent plasma of recovered individuals. Several observational studies have evaluated safety and efficacy of administration of convalescent plasma transfusion in patients with COVID-19 to reduce symptoms and mortality $[82,83]$. The Food and Drug Administration has recognized the clinical effect of investigational convalescent plasma transfusion in patients with serious or immediately life-threatening diseases who lack other therapeutic alternatives.

Tremblay et al. [84] report a case series of 24 patients with cancer (including 1 patient with CLL) who received convalescent plasma as part of an expanded access protocol (NCT04338360). Nonintubated patients, particularly those on nasal cannula alone, had favorable outcomes. Three mild febrile nonhemolytic transfusion reactions were observed. C-reactive protein significantly decreased after 3 days of treatment, while other laboratory parameters including ferritin and $\mathrm{D}$-dimer remained unchanged. After a median of hospital duration of 9 days, 13 patients (54.2\%) had been discharged home, 1 patient (4.2\%) was still hospitalized, and 10 patients had died (41.7\%). This series provides clinical experience of convalescent plasma in patients with COVID-19 who have an underlying malignancy and supports further consideration of convalescent plasma as a therapeutic approach.

\section{The Role of BTKis in SARS-CoV-2 Treatment}

The BTKis ibrutinib and acalabrutinib have been hypothesized to have protective effects against COVID-19 by affecting macrophages and attenuating the immune response leading to weakened hyperinflammatory response and are currently being investigated in the treatment of COVID-19 [71, 85]. Treon et al. [86] reported the impact of ibrutinib in 6 patients with Waldenstrom's macroglobulinemia also infected with COVID-19. The investigators hypothesized that ibrutinib may protect against pulmonary lung injury based on positive results observed in a lethal flu animal model. Five of the 6 patients with Waldenstrom's macroglobulinemia receiving ibrutinib $420 \mathrm{mg} /$ day did not experience dyspnea, required no hospitalization, and showed steady improvement with resolution or near resolution of COVID-19-related symptoms. The sixth patient receiving $140 \mathrm{mg} /$ day experienced progressive dyspnea and hypoxia requiring hospitalization and ultimately recovered after further treatment.

Thibaud et al. [87] reported the outcomes of 8 patients receiving a BTKi (ibrutinib $n=7$ and acalabrutinib $n=1$ ) for CLL and hospitalized with COVID-19. BTKi was held in 6 patients and continued in the remaining two. Two patients in the group that held BTKi developed respiratory failure, and the other patients had mild-to-moderate disease. The 2 patients continuing BTKi treatment had short hospital stays, minimal oxygen requirements, and recovered fully. No significant adverse events attributed to BTKi developed in either patient who continued BTKi therapy.

The authors of both studies concluded that these hypothesis-generating and preliminary results require con- 
firmation from randomized controlled studies to evaluate the benefit-risk profile of ibrutinib in patients hospitalized with COVID-19. The American Society of Hematology (ASH) advises caution with abrupt discontinuation of ibrutinib to avoid a cytokine release syndrome that has been associated with withdrawal [88]. However, as long as there are no prospective data, there is no recommendation to continue BTKi treatment in SARS-CoV-2-infected CLL patients either.

Clinical trials such as iNSPIRE (NCT04375397) or CALAVI (NCT04346199) are evaluating prospectively safety and efficacy of the BTKis ibrutinib or acalabrutinib compared to best supportive care in patients hospitalized with COVID-19-related pulmonary distress. The trials are ongoing, and results are not yet available.

\section{Conclusion}

At this time point, only scarce data on SARS-CoV2-infected CLL patients are available. The following conclusions can be drawn from the published data so far: both treated and untreated CLL patients are at risk for a severe SARS-CoV-2 infection. Advanced age seems to be a risk factor, whereas an ongoing treatment seems to not necessarily affect the course of infection adversely. Among hospitalized patients, the mortality rate is high. BTKis could have a protective effect against severe course of COVID-19 by attenuating hyperinflammatory responses and are currently being investigated in patients hospitalized with COVID-19-related pulmonary distress.

In order to protect patients with CLL from other additional pulmonary complications, every patient should be vaccinated against $S$. pneumoniae and seasonal influenza. To increase the probability of a serological response, vaccination should be as early as possible, particularly before commencement of treatment and onset of hypogammaglobulinemia. To restore the humoral immune system, criteria for immunoglobulin substitution should be evaluated, and immunoglobulins should be substituted if indicated according to EMA guidelines [35].

In asymptomatic CLL patients lacking treatment indication, the watch-and-wait strategy should continue. If the patient is stable, the number of visits should be decreased during pandemic peak to prevent nosocomial SARS-CoV-2 infection. The treating hematologist should consider to replace visits by regular phone calls if blood counts are performed at the general practitioner.

When treatment is indicated as defined by IWCLL guidelines, the treatment should not be postponed, but individual considerations during pandemic may justify a deferral [36]. As proposed by an expert panel, the decision about delivering treatment should be primarily influenced by the tempo of SARS-CoV-2 epidemiology (measured by the reproduction number), the local situation of the hospital, and the potential therapeutic benefit of the antileukemic therapy [89].

Before initiating treatment, SARS-CoV-2 should be excluded by careful examination and PCR testing from nasopharyngeal swabs. The optimal CLL treatment should be chosen based on the individual patients' risk profile including medical history, age, comorbidities, previous lines of treatment, concomitant medication, and genetic and laboratory risk factors, but also on the abovementioned aspects including likely number of visits. Considering treatment-specific side effects, such as B-cell depletion in monoclonal antibodies, neutropenia in chemotherapy and BCL-2 inhibitors, and bacterial and fungal infections in BTKis, is also necessary for treatment recommendation.

However, since there is currently no SARS-CoV-2 vaccine available, the pandemic will continue until further notice. It is important to regularly update the recommendations and if possible to include all CLL patients in clinical trials and/or registries to allow analyzation of the impact of SARS-CoV-2 on the management of patients with CLL.

\section{Conflict of Interest Statement}

P.L. receives research grants from Janssen-Cilag and consulting honoraria from Janssen-Cilag, Abbvie, and Roche. B.E. has received personal fee from Janssen-Cilag, Roche, Novartis, Abbvie, Gilead, Celgene, ArQule, Astrazeneca, Oxford Biomedica, and Adaptive Biotechnologies and research funding from Janssen-Cilag, Roche, Abbvie, Gilead, and Beigene.

\section{Funding Sources}

The authors did not receive any funding.

\section{Author Contributions}

All authors contributed towards patient care, collection and assembly of data, data analysis and interpretation, manuscript writing, and final manuscript approval. 


\section{References}

1 Statistics Sc. 2018. Available from: https:// seer.cancer.gov/statfacts/html/clyl.html.

2 He W, Chen L, Chen L, Yuan G, Fang Y, Chen W, et al. COVID-19 in persons with haematological cancers. Leukemia. 2020;34(6): 1637-45.

3 Tian J, Yuan X, Xiao J, Zhong Q, Yang C, Liu $\mathrm{B}$, et al. Clinical characteristics and risk factors associated with COVID-19 disease severity in patients with cancer in Wuhan, China: a multicentre, retrospective, cohort study. Lancet Oncol. 2020;21(7):893-903.

4 Dai M, Liu D, Liu M, Zhou F, Li G, Chen Z, et al. Patients with cancer appear more vulnerable to SARS-CoV-2: a multicenter study during the COVID-19 outbreak. Cancer Discov. 2020;10(6):783-91.

5 Yang K, Sheng Y, Huang C, Jin Y, Xiong N, Jiang $\mathrm{K}$, et al. Clinical characteristics, outcomes, and risk factors for mortality in patients with cancer and COVID-19 in Hubei, China: a multicentre, retrospective, cohort study. Lancet Oncol. 2020;21(7):904-13.

6 Forconi F, Moss P. Perturbation of the normal immune system in patients with CLL. Blood. 2015;126(5):573-81.

7 Cutucache CE. Tumor-induced host immunosuppression: special focus on CLL. Int Immunopharmacol. 2013;17(1):35-41.

8 Porakishvili N, Kardava L, Jewell AP, Yong K, Glennie MJ, Akbar A, et al. Cytotoxic CD4+ T cells in patients with B cell chronic lymphocytic leukemia kill via a perforin-mediated pathway. Haematologica. 2004;89(4):435-43.

9 Scrivener S, Goddard RV, Kaminski ER, Prentice AG. Abnormal T-cell function in Bcell chronic lymphocytic leukaemia. Leuk Lymphoma. 2003;44(3):383-9.

10 Chiorazzi N, Fu SM, Montazeri G, Kunkel HG, Rai K, Gee T. T cell helper defect in patients with chronic lymphocytic leukemia. J Immunol. 1979;122(3):1087-90.

11 Freeman JA, Crassini KR, Best OG, Forsyth CJ, Mackinlay NJ, Han P, et al. Immunoglobulin $\mathrm{G}$ subclass deficiency and infection risk in 150 patients with chronic lymphocytic leukemia. Leuk Lymphoma. 2013;54(1):99-104.

12 Gale RP, Gale RP, Chapel HM, Bunch C, Rai $\mathrm{KR}$, Foon $\mathrm{K}$, et al. Intravenous immunoglobulin for the prevention of infection in chronic lymphocytic leukemia. A randomized, controlled clinical trial. N Engl J Med. 1988; 319(14):902-7.

13 Sampalo A, Navas G, Medina F, Segundo C, Cámara C, Brieva JA. Chronic lymphocytic leukemia B cells inhibit spontaneous Ig production by autologous bone marrow cells: role of CD95-CD95L interaction. Blood. 2000;96(9):3168-74.

14 Motta M, Chiarini M, Ghidini C, Zanotti C, Lamorgese C, Caimi L, et al. Quantification of newly produced $\mathrm{B}$ and $\mathrm{T}$ lymphocytes in untreated chronic lymphocytic leukemia patients. J Transl Med. 2010;8:111.
15 Kunicka JE, Platsoucas CD. Defective helper function of purified T4 cells and excessive suppressor activity of purified T8 cells in patients with B-cell chronic lymphocytic leukemia. T4 suppressor effector cells are present in certain patients. Blood. 1988;71(6):155160.

16 Kay NE, Perri RT. Evidence that large granular lymphocytes from B-CLL patients with hypogammaglobulinemia down-regulate B-cell immunoglobulin synthesis. Blood. 1989; 73(4):1016-9.

17 Füst G, Miszlay Z, Czink E, Varga L, Pálóczi $\mathrm{K}$, Szegedi G, et al. C1 and C4 abnormalities in chronic lymphocytic leukaemia and their significance. Immunol Lett. 1987;14(3):2559.

18 Manukyan G, Papajik T, Gajdos P, Mikulkova Z, Urbanova R, Gabcova G, et al. Neutrophils in chronic lymphocytic leukemia are permanently activated and have functional defects. Oncotarget. 2017;8(49):84889-901.

19 Kontoyiannis DP, Georgiadou SP, Wierda WG, Wright S, Albert ND, Ferrajoli A, et al. Impaired bactericidal but not fungicidal activity of polymorphonuclear neutrophils in patients with chronic lymphocytic leukemia. Leuk Lymphoma. 2013;54(8):1730-3.

20 Maffei R, Bulgarelli J, Fiorcari S, Bertoncelli L, Martinelli S, Guarnotta C, et al. The monocytic population in chronic lymphocytic leukemia shows altered composition and deregulation of genes involved in phagocytosis and inflammation. Haematologica. 2013;98(7): 1115-23.

21 Huergo-Zapico L, Acebes-Huerta A, Gonzalez-Rodriguez AP, Contesti J, Gonzalez-García E, Payer AR, et al. Expansion of NK cells and reduction of NKG2D expression in chronic lymphocytic leukemia. Correlation with progressive disease. PLoS One. 2014; 9(10):e108326

22 Orsini E, Guarini A, Chiaretti S, Mauro FR, Foa R. The circulating dendritic cell compartment in patients with chronic lymphocytic leukemia is severely defective and unable to stimulate an effective T-cell response. Cancer Res. 2003;63(15):4497-506.

23 Orsini E, Pasquale A, Maggio R, Calabrese E, Mauro FR, Giammartini E, et al. Phenotypic and functional characterization of monocytederived dendritic cells in chronic lymphocytic leukaemia patients: influence of neoplastic CD19 cells in vivo and in vitro. Br J Haematol. 2004;125(6):720-8.

24 Rieger CT, Liss B, Mellinghoff S, Buchheidt D, Cornely OA, Egerer G, et al. Anti-infective vaccination strategies in patients with hematologic malignancies or solid tumors-Guideline of the Infectious Diseases Working Party (AGIHO) of the German Society for Hematology and Medical Oncology (DGHO). Ann Oncol. 2018;29(6):1354-65.
25 Ortqvist A, Hedlund J, Burman LA, Elbel E, Höfer M, Leinonen M, et al. Randomised trial of 23-valent pneumococcal capsular polysaccharide vaccine in prevention of pneumonia in middle-aged and elderly people. Swedish Pneumococcal Vaccination Study Group. Lancet. 1998;351(9100):399-403.

26 Bonten MJ, Huijts SM, Bolkenbaas M, Webber C, Patterson S, Gault S, et al. Polysaccharide conjugate vaccine against pneumococcal pneumonia in adults. N Engl J Med. 2015; 372(12):1114-25.

27 Douglas AP, Trubiano JA, Barr I, Leung V, Slavin MA, Tam CS. Ibrutinib may impair serological responses to influenza vaccination. Haematologica. 2017;102(10):e397-e9.

28 Andrick B, Alwhaibi A, DeRemer DL, Quershi S, Khan R, Bryan LJ, et al. Lack of adequate pneumococcal vaccination response in chronic lymphocytic leukaemia patients receiving ibrutinib. Br J Haematol. 2018;182(5): 712-4.

29 Hartkamp A, Mulder AH, Rijkers GT, van Velzen-Blad H, Biesma DH. Antibody responses to pneumococcal and haemophilus vaccinations in patients with B-cell chronic lymphocytic leukaemia. Vaccine. 2001; 19(13-14):1671-7.

30 Sinisalo $M$, Vilpo J, Itälä $M$, Väkeväinen $M$, Taurio J, Aittoniemi J. Antibody response to 7 -valent conjugated pneumococcal vaccine in patients with chronic lymphocytic leukaemia. Vaccine. 2007;26(1):82-7.

31 Pasiarski M, Rolinski J, Grywalska E, Stelmach-Goldys A, Korona-Glowniak I, Gozdz $\mathrm{S}$, et al. Antibody and plasmablast response to 13-valent pneumococcal conjugate vaccine in chronic lymphocytic leukemia patients: preliminary report. PLoS One. 2014;9(12): e114966.

32 Molica S, Musto P, Chiurazzi F, Specchia G, Brugiatelli M, Cicoira L, et al. Prophylaxis against infections with low-dose intravenous immunoglobulins (IVIG) in chronic lymphocytic leukemia. Results of a crossover study. Haematologica. 1996;81(2):121-6.

33 Jurlander J, Geisler CH, Hansen MM. Treatment of hypogammaglobulinaemia in chronic lymphocytic leukaemia by low-dose intravenous gammaglobulin. Eur J Haematol. 1994;53(2):114-8.

34 Boughton BJ, Jackson N, Lim S, Smith N Randomized trial of intravenous immunoglobulin prophylaxis for patients with chronic lymphocytic leukaemia and secondary hypogammaglobulinaemia. Clin Lab Haematol. 1995;17(1):75-80.

35 EMA. 2018.

36 Hallek M, Cheson BD, Catovsky D, CaligarisCappio F, Dighiero G, Döhner H, et al. iwCLL guidelines for diagnosis, indications for treatment, response assessment, and supportive management of CLL. Blood. 2018;131(25): 2745-60. 
37 Hallek M, Fischer K, Fingerle-Rowson G, Fink AM, Busch R, Mayer J, et al. Addition of rituximab to fludarabine and cyclophosphamide in patients with chronic lymphocytic leukaemia: a randomised, open-label, phase 3 trial. Lancet. 2010;376(9747):1164-74.

38 Fischer K, Bahlo J, Fink AM, Goede V, Herling $\mathrm{CD}$, Cramer $\mathrm{P}$, et al. Long-term remissions after FCR chemoimmunotherapy in previously untreated patients with CLL: updated results of the CLL8 trial. Blood. 2016; 127(2):208-15.

39 Eichhorst B, Fink AM, Bahlo J, Busch R, Kovacs $\mathrm{G}$, Maurer $\mathrm{C}$, et al. First-line chemoimmunotherapy with bendamustine and rituximab versus fludarabine, cyclophosphamide, and rituximab in patients with advanced chronic lymphocytic leukaemia (CLL10): an international, open-label, randomised, phase 3, non-inferiority trial. Lancet Oncol. 2016; 17(7):928-42.

40 Goede V, Fischer K, Busch R, Engelke A Eichhorst B, Wendtner CM, et al. Obinutuzumab plus chlorambucil in patients with CLL and coexisting conditions. N Engl J Med. 2014;370(12):1101-10.

41 Carson KR, Focosi D, Major EO, Petrini M, Richey EA, West DP, et al. Monoclonal antibody-associated progressive multifocal leucoencephalopathy in patients treated with rituximab, natalizumab, and efalizumab: a review from the Research on Adverse Drug Events and Reports (RADAR) Project. Lancet Oncol. 2009;10(8):816-24.

42 Mozessohn L, Chan KK, Feld JJ, Hicks LK Hepatitis B reactivation in HBsAg-negative/ $\mathrm{HBcAb}$-positive patients receiving rituximab for lymphoma: a meta-analysis. J Viral Hepat. 2015;22(10):842-9.

43 Shanafelt TD, Wang XV, Kay NE, Hanson CA, O’Brien S, Barrientos J, et al. Ibrutinibrituximab or chemoimmunotherapy for chronic lymphocytic leukemia. N Engl J Med. 2019;381(5):432-43.

44 Woyach JA, Ruppert AS, Heerema NA, Zhao W, Booth AM, Ding W, et al. Ibrutinib regimens versus chemoimmunotherapy in older patients with untreated CLL. N Engl J Med. 2018;379(26):2517-28.

45 Burger JA, Tedeschi A, Barr PM, Robak T, Owen C, Ghia P, et al. Ibrutinib as initial therapy for patients with chronic lymphocytic leukemia. N Engl J Med. 2015;373(25):2425-37.

46 Byrd JC, Harrington B, O’Brien S, Jones JA, Schuh A, Devereux S, et al. Acalabrutinib (ACP-196) in relapsed chronic lymphocytic leukemia. N Engl J Med. 2016;374(4):323-32.

47 Furman RR, Sharman JP, Coutre SE, Cheson BD, Pagel JM, Hillmen P, et al. Idelalisib and rituximab in relapsed chronic lymphocytic leukemia. N Engl J Med. 2014;370(11):9971007.

48 Sun C, Tian X, Lee YS, Gunti S, Lipsky A, Herman SE, et al. Partial reconstitution of humoral immunity and fewer infections in patients with chronic lymphocytic leukemia treated with ibrutinib. Blood. 2015;126(19):2213-9.
49 Ghez D, Calleja A, Protin C, Baron M, Ledoux MP, Damaj G, et al. Early-onset invasive aspergillosis and other fungal infections in patients treated with ibrutinib. Blood. 2018 131(17):1955-9.

50 Varughese T, Taur Y, Cohen N, Palomba ML, Seo SK, Hohl TM, et al. Serious infections in patients receiving ibrutinib for treatment of lymphoid cancer. Clin Infect Dis. 2018;67(5): 687-92.

51 Kanegane H, Nakano T, Shimono Y, Zhao M, Miyawaki T. Pneumocystis jiroveci pneumonia as an atypical presentation of X-linked agammaglobulinemia. Int J Hematol. 2009; 89(5):716-7.

52 Tisi MC, Hohaus S, Cuccaro A, Innocenti I, De Carolis E, Za T, et al. Invasive fungal infections in chronic lymphoproliferative disorders: a monocentric retrospective study. Haematologica. 2017;102(3):e108-e11.

53 Ahn IE, Jerussi T, Farooqui M, Tian X, Wiestner A, Gea-Banacloche J. Atypical Pneumocystis jirovecii pneumonia in previously untreated patients with CLL on single-agent ibrutinib. Blood. 2016;128(15):1940-3.

54 Ryan CE, Cheng MP, Issa NC, Brown JR, Davids MS. Pneumocystis jirovecii pneumonia and institutional prophylaxis practices in CLL patients treated with BTK inhibitors. Blood Adv. 2020;4(7):1458-63.

55 Dubovsky JA, Beckwith KA, Natarajan G, Woyach JA, Jaglowski S, Zhong Y, et al. Ibrutinib is an irreversible molecular inhibitor of ITK driving a Th1-selective pressure in $\mathrm{T}$ lymphocytes. Blood. 2013;122(15):2539-49.

56 Herman SE, Gordon AL, Hertlein E, Ramanunni A, Zhang X, Jaglowski S, et al. Bruton tyrosine kinase represents a promising therapeutic target for treatment of chronic lymphocytic leukemia and is effectively targeted by PCI-32765. Blood. 2011;117(23):628796.

57 Stiff A, Trikha P, Wesolowski R, Kendra K, Hsu V, Uppati S, et al. Myeloid-derived suppressor cells express Bruton's tyrosine kinase and can be depleted in tumor-bearing hosts by ibrutinib treatment. Cancer Res. 2016; 76(8):2125-36

58 Chamilos G, Lionakis MS, Kontoyiannis DP. Call for action: invasive fungal infections associated with ibrutinib and other small molecule kinase inhibitors targeting immune signaling pathways. Clini Infect Dis. 2018;66(1): $140-8$.

59 Stadler N, Hasibeder A, Lopez PA, Teschner D, Desuki A, Kriege O, et al. The Bruton tyrosine kinase inhibitor ibrutinib abrogates triggering receptor on myeloid cells 1-mediated neutrophil activation. Haematologica. 2017; 102(5):e191-e4.

60 Futatani T, Miyawaki T, Tsukada S, Hashimoto S, Kunikata T, Arai S, et al. Deficient expression of Bruton's tyrosine kinase in monocytes from X-linked agammaglobulinemia as evaluated by a flow cytometric analysis and its clinical application to carrier detection. Blood. 1998;91(2):595-602.
61 Fiedler K, Sindrilaru A, Terszowski G, Kokai E, Feyerabend TB, Bullinger L, et al. Neutrophil development and function critically depend on Bruton tyrosine kinase in a mouse model of X-linked agammaglobulinemia. Blood. 2011;117(4):1329-39.

62 Perkhofer S, Kehrel BE, Dierich MP, Donnelly JP, Nussbaumer W, Hofmann J, et al. Human platelets attenuate Aspergillus species via granule-dependent mechanisms. J Infect Dis. $2008 ; 198(8): 1243-6$

63 Seymour JF, Kipps TJ, Eichhorst B, Hillmen P, D'Rozario J, Assouline S, et al. Venetoclaxrituximab in relapsed or refractory chronic lymphocytic leukemia. N Engl J Med. 2018; 378(12):1107-20.

64 Roberts AW, Davids MS, Pagel JM, Kahl BS, Puvvada SD, Gerecitano JF, et al. Targeting BCL2 with venetoclax in relapsed chronic lymphocytic leukemia. N Engl J Med. 2016; 374(4):311-22.

65 Fischer K, Al-Sawaf O, Bahlo J, Fink A-M Tandon M, Dixon M, et al. Venetoclax and obinutuzumab in patients with CLL and coexisting conditions. N Engl J Med. 2019; 380(23):2225-36.

66 Leverson JD, Phillips DC, Mitten MJ, Boghaert ER, Diaz D, Tahir SK, et al. Exploiting selective BCL-2 family inhibitors to dissect cell survival dependencies and define improved strategies for cancer therapy. Sci Transl Med. 2015;7(279):279ra40.

67 Stilgenbauer S, Eichhorst B, Schetelig J, Coutre S, Seymour JF, Munir T, et al. Venetoclax in relapsed or refractory chronic lymphocytic leukaemia with $17 \mathrm{p}$ deletion: a multicentre, open-label, phase 2 study. Lancet Oncol. 2016;17(6):768-78

68 Davids MS, Hallek M, Wierda W, Roberts AW, Stilgenbauer S, Jones JA, et al. Comprehensive safety analysis of venetoclax monotherapy for patients with relapsed/refractory chronic lymphocytic leukemia. Clin Cancer Res. 2018;24(18):4371-9.

69 de Weerdt I, Hofland T, de Boer R, Dobber JA, Dubois J, van Nieuwenhuize D, et al. Distinct immune composition in lymph node and peripheral blood of CLL patients is reshaped during venetoclax treatment. Blood Adv. 2019;3(17):2642-52.

70 Cairo MS, Bishop M. Tumour lysis syndrome: new therapeutic strategies and classification. Br J Haematol. 2004;127(1):3-11.

71 Scarfò L, Chatzikonstantinou T, Rigolin GM, Quaresmini G, Motta M, Vitale C, et al. COVID-19 severity and mortality in patients with chronic lymphocytic leukemia: a joint study by ERIC, the European Research Initiative on CLL, and CLL Campus. Leukemia. 2020:1-10.

72 Mato AR, Roeker LE, Lamanna N, Allan JN, Leslie L, Pagel JM, et al. Outcomes of COVID-19 in patients with CLL: a multicenter international experience. Blood. 2020; 136(10):1134-43. 
73 Paneesha S, Pratt G, Parry H, Moss P. Covid-19 infection in therapy-naive patients with B-cell chronic lymphocytic leukemia. Leuk Res. 2020;93:106366.

74 Fürstenau M, Langerbeins P, De Silva N, Fink AM, Robrecht S, von Tresckow J, et al. COVID-19 among fit patients with CLL treated with venetoclax-based combinations. Leukemia. 2020;34(8):2225-9.

75 Martín-Moro F, Marquet J, Piris M, Michael BM, Sáez AJ, Corona M, et al. Survival study of hospitalised patients with concurrent COVID-19 and haematological malignancies. $\mathrm{Br}$ J Haematol. 2020;190(1):e16-20.

76 Baumann T, Delgado J, Montserrat E. CLL and COVID-19 at the Hospital Clinic of Barcelona: an interim report. Leukemia. 2020; 34(7):1954-6.

77 Reda G, Noto A, Cassin R, Zamprogna G, Borella C, Scarfò L, et al. Reply to "CLL and COVID-19 at the Hospital Clinic of Barcelona: an interim report" Analysis of six hematological centers in Lombardy: on behalf of CLL commission of Lombardy Hematology Network (REL). Leukemia. 2020;34(9):25312.

78 Beigel JH, Tomashek KM, Dodd LE, Mehta AK, Zingman BS, Kalil AC, et al. Remdesivir for the treatment of Covid-19: preliminary report. N Engl J Med. 2020 Nov 5;383(19): 1813-26.
79 Horby P, Lim WS, Emberson JR, Mafham M, Bell JL, Linsell L, et al. Dexamethasone in hospitalized patients with covid-19: preliminary report. N Engl J Med. 2020.

80 Kuderer NM, Choueiri TK, Shah DP, Shyr Y, Rubinstein SM, Rivera DR, et al. Clinical impact of COVID-19 on patients with cancer (CCC19): a cohort study. Lancet. 2020; 395(10241):1907-18.

81 Rivera DR, Peters S, Panagiotou OA, Shah DP, Kuderer NM, Hsu CY, et al. Utilization of COVID-19 treatments and clinical outcomes among patients with cancer: a COVID-19 and Cancer Consortium (CCC19) cohort study. Cancer Discov. 2020;10(10):1514-27.

82 Hegerova L, Gooley TA, Sweerus KA, Maree C, Bailey N, Bailey M, et al. Use of convalescent plasma in hospitalized patients with COVID-19: case series. Blood. 2020;136(6):75962.

83 Zeng QL, Yu ZJ, Gou JJ, Li GM, Ma SH, Zhang GF, et al. Effect of convalescent plasma therapy on viral shedding and survival in patients with coronavirus disease 2019. J Infect Dis. 2020;222(1):38-43.
84 Tremblay D, Seah C, Schneider T, Bhalla S, Feld J, Naymagon L, et al. Convalescent plasma for the treatment of severe COVID-19 infection in cancer patients. Cancer Med. 2020 Nov;9(22):8571-8.

85 NG J, Hams E, Smith S, Wynne C, Byrne JC, Brennan $\mathrm{K}$, et al. Btk regulates macrophage polarization in response to lipopolysaccharide. PloS one. 2014;9(1):e85834.

86 Treon SP, Castillo JJ, Skarbnik AP, Soumerai JD, Ghobrial IM, Guerrera ML, et al. The BTK inhibitor ibrutinib may protect against pulmonary injury in COVID-19-infected patients. Blood. 2020;135(21):1912-5.

87 Thibaud S, Tremblay D, Bhalla S, Zimmerman B, Sigel K, Gabrilove J. Protective role of Bruton tyrosine kinase inhibitors in patients with chronic lymphocytic leukaemia and COVID-19. British journal of haematology. 2020 Jul;190(2):e73-76.

88 A.S.o. COVID-19 and CLL: frequently asked questions. Hematology. 2020; COVID-19 Resources. Available from: https://hematology. org/covid-19/covid-19-and-cll.

89 Rossi D, Shadman M, Condoluci A, Brown JR, Byrd JC, Gaidano G, et al. How we manage patients with chronic lymphocytic leukemia during the SARS-CoV-2 pandemic. HemaSphere. 2020;4(4):e432. 